\title{
Chaos and Dynamical Systems
}

\author{
by
}

\author{
Khristine M. Krcelic
}

Submitted in Partial Fulfillment of the Requirements

for the Degree of

Master of Science

in the

Mathematics

Program

YOUNGSTOWN STATE UNIVERSITY

December, 2012

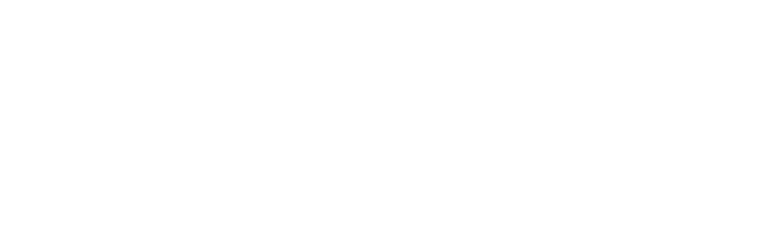




\title{
Chaos and Dynamical Systems
}

\author{
Khristine M. Krcelic
}

I hereby release this thesis to the public. I understand that this thesis will be made available from the OhioLINK ETD Center and the Maag Library Circulation Desk for public access. I also authorize the University or other individuals to make copies of this thesis as needed for scholarly research.

Signature:

Khristine M. Krcelic, Student

Date

Approvals:

Eric Wingler, Thesis Advisor

Date

Zbigniew Piotrowski, Committee Member

Date

Jamal Tartir, Committee Member

Date

Peter J. Kasvinsky, Dean of School of Graduate Studies \& Research Date 
Khristine M. Krcelic

2012 


\begin{abstract}
Throughout the ages, mathematics has been evolving and creating new branches. In the middle to late twentieth century, a new branch formed: chaos. Chaos is the study of dynamical systems that vary greatly with respect to initial conditions. The slightest change in an initial condition, a seemingly unnoticeable change, can yield a drastically different result if the system is chaotic. Hence the common term relating to chaos theory, "the butterfly effect". Something as minute as the flap of a butterfly's wings could spawn a natural disaster half-way across the world. This thesis provides an insight to chaos from both a pure and an applied mathematician's point of view.
\end{abstract}




\section{Contents}

1 Introduction 1

2 From the Perspective of a Pure Mathematician 3

3 From the Perspective of the Applied Mathematician 13

4 Conclusion $\quad 17$

$\begin{array}{llr}5 & \text { Bibliography } & 18\end{array}$ 


\section{Introduction}

"Many people believe that twentieth century science will be remembered for three main theories: quantum mechanics, relativity, and chaos. Chaos theory is a blanketing theory that covers all aspects of science, hence, it shows up everywhere in the world today: mathematics, physics, biology, finance, and even music. Where classical sciences end, chaos is only beginning" [3].

According to Dictionary.com [4], chaos theory is the study of unpredictable and complex dynamic systems that are highly sensitive to small changes in external conditions. So what does that mean in English? Let's look at an example.

A bread maker will knead their dough until it is ready to be left to rise. Now, consider two raisins that were placed in the dough near each other. As the bread maker kneads the bread, he folds and then flattens the dough and repeats the process over and over again. The first few times he goes through the process, the raisins might stay the same distance apart from one another. This could lead to the thought that this is not a chaotic system and that the raisins will stay the same distance apart throughout the entire kneading process. However, after a few more times going through the kneading process the raisins eventually begin to separate farther and farther from one another and eventually into different parts of the folding process. One might think that eventually the raisins will regain their initial positions, hence making the system periodic and not chaotic.

So, why does the bread maker knead the dough?

The answer is... "to mix up the ingredients ... If you mix stuff up, the individual particles have to move in a very irregular way. Particles that start close together end up far apart; points far apart may be folded back to be close together. In short, chaos 
is the natural result of mixing" [24]. When we think of chaos as a result of mixing, it can be applied to many things in life, not just to the mathematical and scientific world but also the physical world. 


\section{From the Perspective of a Pure Mathematician}

Robert Devaney cites in Chaos and Fractals: The Mathematics Behind the Computer Graphics [9] a formal definition for chaos:

Definition 1 (Chaos). Let $f: M \rightarrow M$ be a map, where $M$ is a metric space. The map $f$ is said to be chaotic if

1. $f$ has sensitive dependence on initial conditions,

2. $f$ is topologically transitive, and

3. Periodic points of $f$ are dense in $M$.

This is the definition that will be referred to in this paper. But let's break this down.

Definition 2 (Sensitive Dependence). A map $f$, having sensitive dependence on initial conditions, implies that "small errors in choosing initial points for orbits may lead to vast errors along the resulting orbits" [15]. A technical definition using quantifiers would be There exists $\delta>0$, such that for all $x \in[0,1]$, there exists a $y \in I$ and $n \in \mathbb{N}$ such that $\left|f^{n}(x)-f^{n}(y)\right| \geq \delta$ where $I$ denotes an open-in-[0,1] interval [10].

Definition 3 (Topologically Transitive). A map $f$ is topologically transitive if given any two intervals $U$ and $V$, there is some positive integer $k$ such that $f^{k}(U) \cap V \neq \emptyset$ [26].

Finally, the periodic points that are dense allow for an element of regularity.

In order to look at examples, we need to look at fractals. "Certain sets of equations...can be repeated many times, creating images called fractals...(The) fractals 
exhibit all of the chaotic traits": sensitivity to small changes, unpredictability with a sense of regularity [2]. We will be taking the equation given and repeating it many times, also known as iterating the equation.

The first example we'll look at is the Doubling Function.

Theorem 1 (The Doubling Function is Chaotic). The map $f: S^{1} \rightarrow S^{1}$ given by $f(\theta)=2 \theta(\bmod 2 \pi)$ is chaotic.

Proof. Note that $f(\theta)$ is not well-defined, we can make it well-defined by noting that $f(\theta)=2 \theta-2 \pi\left\lfloor\frac{\theta}{\pi}\right\rfloor$. Note that $S^{1}$ is the unit circle on the plane and $\theta$ is given in radians. Since $\theta$ is doubled upon iteration in the function $f, f$ is sensitive to initial conditions. The $\operatorname{arc}$ on $S^{1}$, associated with $\theta$, is doubled upon iteration in the function $f$. So eventually upon some iteration $k$, the arc will cover all of $S^{1}$ and in particular any other arc of $S^{1}$. Thus, $f$ is topologically transitive. A point is determined by any angle of the form $\theta+2 k \pi, k \in \mathbb{Z}$. So $\theta$ is a periodic point of period $n$ if and only if $2 n \theta=\theta+2 k \pi, k \in \mathbb{Z}$. Therefore, the doubling map is chaotic.

Sierpinski's Triangle is a famous example of a fractal. To begin, one starts with an equilateral triangle. Then in the first iteration, the middle triangle of the quarteredtriangle is removed. In the next iteration, and the iterations to follow, remove the inner triangle of the leftover quartered-triangles. In doing so, the following fractal is created.

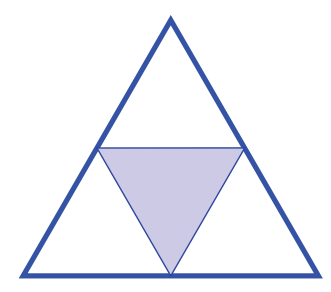


If you'd like to look at, or create, this fractal in a less picturesque way, here is how a computer would handle generating the Sierpinski's Triangle.

First, an initial point $(x, y)$ is chosen. Then, the program would come up with a random number between 0 and 1 . If the number is between 0 and $\frac{1}{3}$, then the following equations would be used to create the next point to be iterated:

$$
\begin{gathered}
x_{n}=\frac{1}{2}\left(x_{n-1}+1\right) \\
\text { and } \\
y_{n}=\frac{1}{2} y_{n-1} .
\end{gathered}
$$

If the random number that was chosen is between $\frac{1}{3}$ and $\frac{2}{3}$, then we'd use the equations:

$$
\begin{gathered}
x_{n}=\frac{1}{2} x_{n-1} \\
\text { and } \\
y_{n}=\frac{1}{2} y_{n-1} .
\end{gathered}
$$

Finally, if the number chosen at random was between $\frac{2}{3}$ and 1 , we would use the equations: 


$$
x_{n}=\frac{1}{2}\left(x_{n-1}+\frac{1}{2}\right)
$$

and

$$
y_{n}=\frac{1}{2}\left(y_{n-1}+1\right) \text {. }
$$

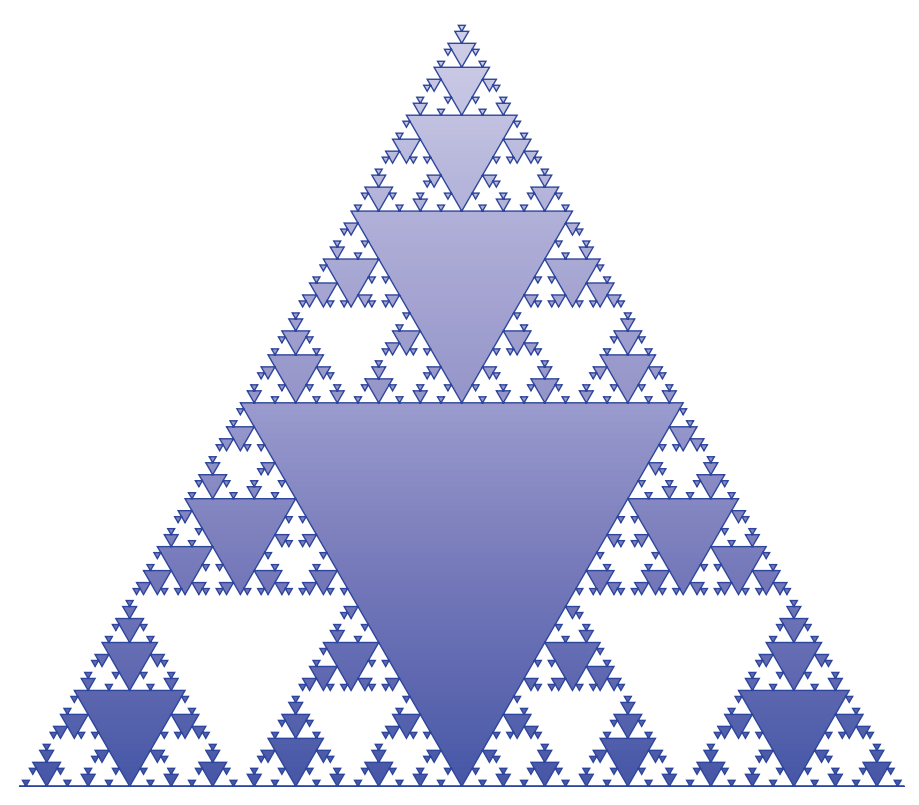

Regardless of which way Sierpinski's Triangle is approached, the result is the same. Another famous fractal is the Koch Snowflake. As in Sierpinski's Triangle, we once again begin with a triangle. However, instead of removing the inner triangle of the quartered-triangle, we remove the middle third of each side of the triangle and add two sides of equal length. We then continue this process until we achieve the Koch Snowflake. "The ultimate result is a curve that is infinitely wiggly - there are no straight lines in it whatsoever" [14]. 

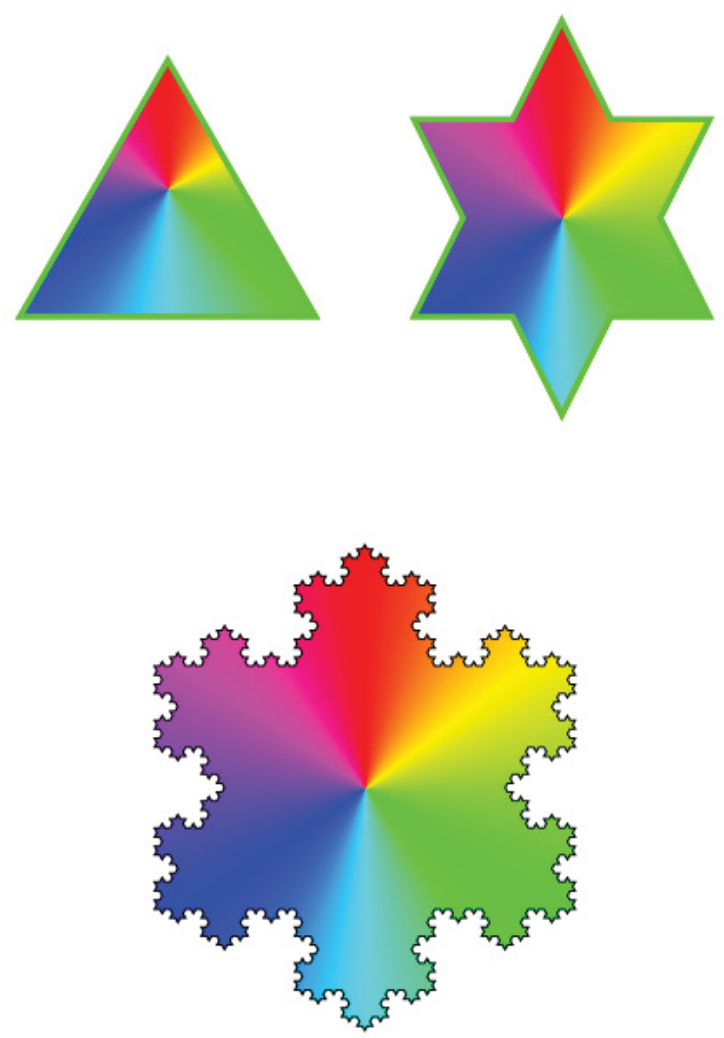

Being a "wiggle" is another way to be chaotic. Having a wiggle or woggle tendency is something addressed and looked at in depth in John Banks, Valentina Dragan, and Arthur Jones' Chaos: A Mathematical Introduction [10]. A definition we will need to understand the concept of a wiggle or woggle is that of a hump mapping.

Definition 4 (One-hump Mapping). A mapping $f$ is a one-hump mapping on $[a, c]$ if it is continuous and there exist $b$ in $(a, c)$ such that $f$ is strictly increasing on $[a, b]$ and strictly decreasing on $[b, c]$.

Definition 5 (m-hump mapping). The mapping $f$ is an m-hump mapping on $[0,1]$ if there are $m+1$ points,

$$
0=x_{0}<x_{1}<\ldots<x_{m}=1
$$


such that $f$ is a one-hump mapping on each interval $\left[x_{i-1}, x_{i}\right]$. The interval $\left[x_{i-1}, x_{i}\right]$ is called the base of the $i^{\text {th }}$ hump of $f$.

Definition 6 (Wiggle). A mapping $f$ is a map with wiggly iterates if $f^{n}$ is a $2^{n-1}$ -hump mapping, for each $n \geq 1$, and the length of the largest base of the humps of $f^{n}$ tends to 0 as $n$ tends to infinity.

Definition 7 (Woggle). Assuming that a map $f$ has a derivative, a woggle is a hump of the derivative mapping of $f$ (the hump is in the map of $f^{\prime}$ ) whose base contains an interval $I$ and a point $a \in I$ such that

i. $f^{\prime}>0$ on $I$ and $f^{\prime}$ has a positive local minimum at a,

or

ii. $f^{\prime}<0$ on $I$ and $f^{\prime}$ has a negative local maximum at a.

A lemma that will demonstrate an example of a wiggly iterate is the Spike Lemma.

Theorem 2 (Spike Lemma). Let $f:[0,1] \rightarrow[0,1]$ have wiggly iterates. For every interval $I \subseteq[0,1]$ there is a hump of some $f^{n}$ whose base is contained in $I$.

Proof. Now, $f$ having wiggly iterates implies by definition that the length of the largest base of the humps of $f^{n}$ tends to 0 as $n$ tends to infinity. Thus we can choose an $n$ such that the distance between two consecutive zeroes of $f^{n}$ is less than half the length of $I$. By choosing the smallest zero that lies in $I$, call it $x_{i}$, we can guarantee that $x_{i}$ is in the first half of the interval. Then the next zero $x_{i+1}$ of $f^{n}$ will be lying in the interval $I$. Hence $\left[x_{i}, x_{i+1}\right] \subseteq I$.

Now, based off of the Spike Lemma, Theorem 2, we can now conclude the following theorems. 


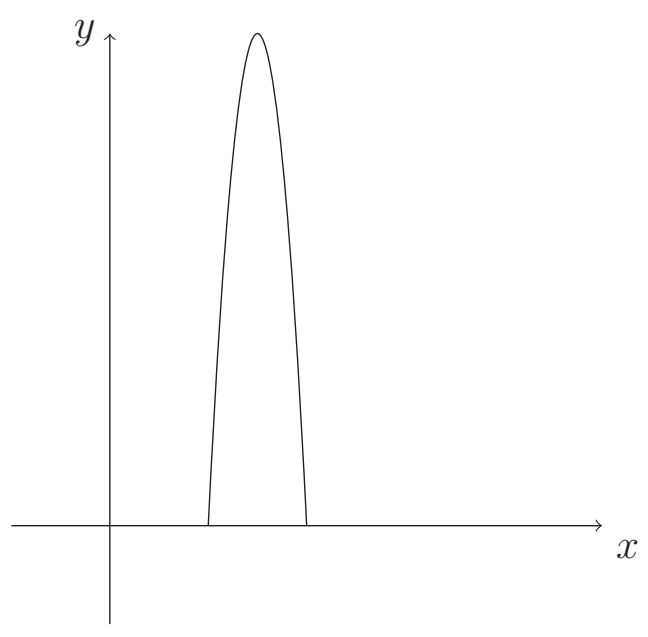

Theorem 3 (Wiggly implies Sensitive). If $f:[0,1] \rightarrow[0,1]$ has wiggly iterates, then $f$ has sensitive dependence everywhere with sensitivity constant $\frac{1}{2}$.

Proof. Suppose a mapping $f$ has wiggly iterates. It will be shown that $f$ has sensitive dependence at all points through definition 2 . Since we will show that the sensitivity constant is $\frac{1}{2}$ we shall let $\delta=\frac{1}{2}$. Let $x$ be any point in $[0,1]$, and let $I$ be any openin- $[0,1]$ interval containing $x$. Now, we need to show the existence of a $y \in I$ and $n \in \mathbb{N}$ such that $\left|f^{n}(x)-f^{n}(y)\right| \geq \frac{1}{2}$. By the Spike Lemma, Theorem 2, there is an $n$ such that for the mapping $f^{n}$, the base of some hump is contained in $I$. If $f^{n}(x) \leq \frac{1}{2}$, then choose $y \in I$ such that $f^{n}(y)=1$. If $f^{n}(x)>\frac{1}{2}$, then choose $y \in I$ such that $f^{n}(y)=0$. Either way, there exists a $y \in I$ such that $\left|f^{n}(x)-f^{n}(y)\right| \geq \frac{1}{2}$ is satisfied. Thus the theorem is proved.

We shall now tackle the topologically transitive feature of a chaotic system.

Theorem 4 (Wiggly implies Transitivity). If $f:[0,1] \rightarrow[0,1]$ has wiggly iterates then $f$ is transitive. 
Proof. Suppose a mapping $f$ has wiggly iterates and let $U$ and $V$ be arbitrary subintervals of $[0,1]$. We want to show that there is an $n \in \mathbb{N}$ such that $f^{n}(U) \bigcap V \neq \emptyset$. By the Spike Lemma, Theorem 2, there is an $n$ such that for the mapping $f^{n}$, the base of some hump is contained in $U$. So, for this $n$ we have that $f^{n}(U)=[0,1]$. Thus $f^{n}(U) \cap V \neq \emptyset$ and so $f$ is transitive and the theorem is proved.

Finally, we need to satisfy the dense periodic points part of the chaotic definition.

Theorem 5 (Wiggly implies Dense Periodic Points). If $f:[0,1] \rightarrow[0,1]$ has wiggly iterates, then the set of all periodic points of $f$ is dense in the interval $[0,1]$.

Proof. Suppose a mapping $f$ has wiggly iterates. We need to show that the set of all periodic points of $f$ is dense in the interval $[0,1]$. In other words, we need to show that every interval $I \subseteq[0,1]$ contains a periodic point of $f$. Let $I$ be any subinterval of $[0,1]$. By the Spike Lemma, Theorem 2, there is an $n$ such that for the mapping $f^{n}$, the base of some hump is contained in $I$. The graph of this hump-mapping must intersect the identity graph $(f(x)=x)$ in at least two points by the Intermediate Value Theorem. When we project these points onto the domain, we obtain at least two periodic points of $f^{n}$. Thus $f^{n}$ has at least two periodic points in $I$.

By putting together the three previous theorems Theorem 3, Theorem 4, Theorem 5 , we can see that wiggly implies chaotic.

Another example of a function that is chaotic is the logistic mapping. The logistic function is $f_{\mu}(x)=\mu x(1-x)$.

Proposition 1. The logistic function has the following properties:

i. $f_{\mu}(0)=f_{\mu}(1)=0$ and $f_{\mu}\left(p_{\mu}\right)=p_{\mu}$, where $p_{\mu}=\frac{\mu-1}{\mu}$ 
ii. $0<p_{\mu}<1$ if $\mu>1$

Proof. Ad (i.)

$$
\begin{aligned}
f_{\mu}(0) & =\mu(0)(1-0)=0 \\
f_{\mu}(1) & =\mu(1)(1-1)=0 \\
f_{\mu}\left(p_{\mu}\right) & =f_{\mu}\left(\frac{\mu-1}{\mu}\right) \\
& =\mu\left(\frac{\mu-1}{\mu}\right)\left(1-\frac{\mu-1}{\mu}\right) \\
& =(\mu-1)\left(\frac{\mu-\mu+1}{\mu}\right) \\
& =(\mu-1) \frac{1}{\mu} \\
& =p_{\mu}
\end{aligned}
$$

Ad (ii.) If $\mu>1$, then $p_{\mu}=\frac{\mu-1}{\mu}>0$ since $\mu-1>0$ and clearly $p_{\mu}=\frac{\mu-1}{\mu}<1$ since $\mu-1<\mu$.

A specific example of the logistic function is a $f_{4}$.

Proposition 2. The logistic function at $\mu=4, f_{4}(x)=4 x(1-x)$, is chaotic on the interval $[0,1]$.

Proof. Let $g(\theta)=2 \theta$ such that $g: S^{1} \rightarrow S^{1}$. Define $h_{1}: S^{1} \rightarrow[-1,1]$ by $h_{1}(\theta)=$ $\cos (\theta)$ (hence projecting $S^{1}$ onto the x-axis). Let $q(x)=2 x^{2}-1$. Then 


$$
\begin{aligned}
h_{1} \circ g(\theta) & =\cos (2 \theta) \\
& =2 \cos ^{2}(\theta)-1 \\
& =q \circ h_{1}(\theta)
\end{aligned}
$$

So $h_{1}$ conjugates $g$ with $q$. Thus $q$ is topologically conjugate to $f_{4}$. Let $h_{2}: S^{1} \rightarrow$ $[-1,1]$. If $h_{2}(t)=\frac{1}{2}(1-t)$, then we have $f_{4} \circ h_{2}=h_{2} \circ q$. And so $f_{4}$ is topologically transitive, for if $U$ and $V$ are two open intervals in $[0,1]$, choose open arcs $U^{\prime}$ and $V^{\prime}$ in $S^{1}$ which project onto $U$ and $V$ under $h_{2} \circ h_{1}$. Since there is a $k$ such that $g^{k}\left(U^{\prime}\right) \bigcap V^{\prime} \neq \emptyset$, we therefore have $f_{4}^{k}(U) \bigcap V \neq \emptyset$. To show sensitivity on initial conditions, we will start by noting that any neighborhood of $U$ of $x \in[0,1]$ "lifts" to $U^{\prime}$ in $S^{1}$. So there is an $n$ such that $g^{n}\left(U^{\prime}\right)$ covers $S^{1}$, so $f_{4}^{n}(U)$ covers $[0,1]$ as well. Hence there are points in $U$ which move at least $\delta=\frac{1}{2}$ away from $x$. To show the density of periodic points, we will note the function $g$ is chaotic and hence has periodic points that are dense. Therefore, there exists $g$-periodic points in $U^{\prime}$. The projection of this point in $U$ is clearly $f_{4}$ periodic.

Thus, there are many applications for a pure mathematician in the relatively new field of chaos. 


\section{From the Perspective of the Applied Mathemati- cian}

"The Butterfly Effect" is probably the most recognizable application for chaos theorists. The term was coined by Edward Lorenz in the mid-twentieth century. Lorenz was an American mathematician and meteorologist. The combination of the two professions led him to become interested in chaos theory in order to better predict the weather. While trying to find a way to better predict the weather, Lorenz determined twelve equations that would help him become more accurate. The twelve equations modeled the weather. Lorenz would utilize a computer to examine the outputs that different inputs would yield. He found that the system evolved differently based on a slight difference in input. This was discovered when he was trying to further analyze a sequence. However, instead of starting at the same initial point, he decided to input a number from the middle of the sequence to cut back on waiting time. When the computer was done computing Lorenz realized a rather puzzling error in the computations. The results were not the same but drastically different. It took Lorenz some time to notice that the original number was stored in the computer out to the millionths place but the number he entered was only out to the thousandths place. This led to his curiosity into the value of sensitive dependence. This in turn would lead to the popular term today of "The Butterfly Effect".

Why "The Butterfly Effect"? Lorenz noticed that the slightest change in the error of the air velocity would result in a change in the original weather pattern. This slightest change in air velocity could be credited to the flap of a butterfly's wings.

He eventually whittled the twelve equations down to three more manageable equa- 
tions that would exhibit a similar behavior. The three equations were

$$
\begin{gathered}
\frac{d x}{d t}=\sigma(y-x) \\
\frac{d y}{d t}=r x-y-x z \\
\frac{d z}{d t}=x y-b z .
\end{gathered}
$$

The dependent variables are $\mathrm{x}, \mathrm{y}$, and $\mathrm{z}$, while $\mathrm{t}$ denotes time, and $\sigma, \mathrm{r}$, and $\mathrm{b}$ are the parameters. While these three equations were not demonstrative of weather patterns, they were demonstrative of another type of chaotic system. These equations effectively modeled a leaky waterwheel, "The Lorenzian Waterwheel". The Lorenzian waterwheel was "the first, famous chaotic system discovered by Edward Lorenz" [16]. Imagine a waterwheel made of cups with holes in the bottom. Water entering the cups at a steady rate is not enough to determine the motion of the wheel. If the water entering the cup is being poured too slowly, then the cup (remembering the hole in the bottom) will never fill up and the waterwheel will never be set in motion. If the water entering the cup is being poured at the correct flow, then the cups will fill and empty at the proper speed and the wheel will be set into its normal motion. However, if the water entering the cup is being poured too fast, then the cup will not have enough time to drain properly and the wheel will either stop or reverse its motion. Under these equations, the waterwheel's motions can never repeat itself nor will it ever become predictable.

Another example in nature where chaos is involved is in the shorelines. The shorelines or coastlines themselves are an example of a fractal and hence an example of chaos in geology. The shores from above look like a long wiggly line that provides 
a barrier between the land and the sea. However, if you zoom in on the coastlines, into the bays and into the coves, you would eventually see something that resembles the aforementioned Koch Snowflake. On the shorelines usually lie numerous varieties of seashells that exhibit chaotic characteristics. Let's look specifically at cone shells. "Some cone shells have what seem to be random collections of triangles of various sizes" [23]. These triangles could be attributed to fractals, which are related to dynamical chaos. Dynamical chaos is an irregular behavior that occurs in a deterministic mathematical system. Hence, "the cone shell combines mathematical features of order and chaos in one pattern" [23].

The impacts of chaos theory are also seen in the human body. The way our veins twist and weave themselves inside our bodies is an example of a fractal, previously mentioned, and hence chaotic. Another body part whose behavior can be modeled by chaos theory is the human heart, in particular the human heartbeat. Not just with a normal heartbeat, but chaos theory presents itself when a patient is in fibrillation, or an irregular beating of the heart. "Traditionally, thoughts about fibrillation took two forms. One classic idea was that secondary pacemaking signals come from abnormal centers within the heart muscle itself, conflicting with the main signal". The other approach focused not on the initiation of electrical waves but on the way they are conducted geographically through the heart" [Harvard-M.I.T. researchers] found that abnormalities in the wave, spinning in tight circles, could cause "re-entry, in which some areas begin a new beat too soon, preventing the heart from pausing for the quiet interval necessary to maintain coordinated pumping"[16]. Either of the approaches shows an awareness to a small change in a parameter. The parameter here is the timing or electrical conductivity. A slight change in this parameter can push an otherwise healthy heart into an unhealthy situation such as a heart attack or sudden 
death.

There are other systems in the human body whose behavior can be explained using chaos theory. In the respiration system, breathing disorders such as panting, sighing, and Cheyne-Stokes respiration, which is a "breathing characterized by rhythmic waxing and waning of the depth of respiration" which could lead to heart failure and brain damage [5]. SIDS, or Sudden Infant Death Syndrome in which an infant under the age of one year dies from unexplained reasons, is another example of chaos in humans [1]. Blood disorders, such as forms of leukemia, which can disrupt or alter the "balance of white and red cells, platelets and lymphocytes" are examples which can be explained by chaos theory. Also, brain disorders like schizophrenia and depression are examples of chaos. These disorders can be related to chaos behavior since a small difference in the brains' fluid-filled cavities can lead to schizophrenia and chemical imbalances in the brain can lead to depression [6],[7]. Both disorders are a slight variation of a healthy brain that leads to dramatically different results (healthy human versus unhealthy human).

Hence, there are numerous applications for an applied mathematician to look at in the field of chaos. 


\section{Conclusion}

"The discovery of chaos required many things and many people. It needed pure mathematicians to develop the topological approach to qualitative dynamics, and to ask sufficiently general questions. It needed physicists to link the answers to the real world. It needed experimentalists to check that the theories made sense. It needed electronic engineers to design and build computers with good graphics and powerful number-crunching capabilities ... It's the combinations that [allows the discovery to work]" [21]. Chaos is found not only in the pure mathematics world but also in the applied world of mathematics. It can be realized by not only those trained in mathematics but also to the person that may not be as mathematically inclined. It is a theory that has been present in the world since the beginning but has just recently been looked at as a science. 


\section{Bibliography}

\section{References}

[1] American sids institute. http://sids.org/nprevent.htm, 2012.

[2] Chaos \& fractals. http://library.thinkquest.org/3703/, 2012.

[3] Chaos theory. http://library.thinkquest.org/3493/noframes/chaos.html, 2012.

[4] Dictionary.com. http://dictionary.reference.com/browse/chaos+theory? $\mathrm{s}=\mathrm{t}, 2012$.

[5] The free dictionary by farlex. http://medical-dictionary . thefreedictionary.com/Cheyne-Stokes+respirations, 2012.

[6] National institute of mental health: Schizophrenia. http://www.nimh.nih.gov/ health/publications/schizophrenia/complete-index.shtml, 2012.

[7] Webmd: Depression. http://www.webmd.com/depression/default.htm, 2012.

[8] Sauer T. D. \& Yorke J. A. Alligood, K. T. Chaos: An Introduction to Dynamical Systems. Springer-Verlag New York, Inc., New York, New York, 1997.

[9] Yorke J. A. et al. Alligood, K. T. Chaos and Fractals: The Mathematics Behind the Computer Graphics, volume 39. American Mathematical Society, Providence, Rhode Island, 1980.

[10] A. Banks, J. Dragan V. \& Jones. Chaos: A Mathematical Introduction. Press Syndicate of the University of Cambridge, New York, New York, 2003. 
[11] F. D. Briggs, J. \& Peat. Turbulent Mirror: An Illustrated Guide to Chaos Theory and the Science of Wholeness. Harper \& Row Publishing, New York, New York, 1989.

[12] J. Briggs. Fractals: The Patterns of Chaos. Touchstone, New York, New York, 1992.

[13] M. W. \& Smale S. Devaney, R. L. Hirsh. Differential Equations, Dynamical Systems, \& An Introduction to Chaos. Elsevier, Singapore, 2 edition, 2004.

[14] R. Devaney. Chaos, Fractals, and Dynamics: Computer Experiments in Mathematics. Addison-Wesley Publishing Company, Menlo Park, California, 1990.

[15] R. L. Devaney. An Introduction to Chaotic Dynamical Systems. Addison-Wesley Publishing Company, Redwood City, California, 2 edition, 1989.

[16] J. Gleick. Chaos: Making a New Science. Penguin Books, New York, New York, 2008.

[17] R. Lewin. Life at the Edge of Chaos. Macmillan Publishing Company, New York, New York, 1992.

[18] I. \& Toffler A. Prigogine, I. Stergers. Order Out of Chaos: Man's New Dialogue with Nature. Shambhala Publications, Boulder, Colorado, 1984.

[19] G. E. Slethaug. Beautiful Chaos: Chaos Theory and Metachaotics in Recent American Fiction. State University of New York, Albany, New York, 2000.

[20] R. Solomon. The Little Book of Mathematical Principles, Theories 8 Things. Metro Books, New York, New York, 2008. 
[21] I. Stewart. Does God Play Dice?: The New Mathematics of Chaos. Blackwell Publishing, Malden, Massachusetts, 2002.

[22] I. Stewart. The Story of Mathematics: From Babylonian Numerals to Chaos Theory. Quercus Publishing Plc., Bloomsbury Square, London, 2008.

[23] I. Stewart. The Mathematics of Life. Basic Books, New York, New York, 2011.

[24] I. Stewart. In Pursuit of the Unknown: 17 Equations that Changed the World. Basic Books, New York, New York, 2012.

[25] M. Ward. Beyond Chaos: The Underlying Theory Behind Life, the Universe, and Everything. Thomas Dunne Books, New York, New York, 2001.

[26] E. W. Weisstein. Topologically transitive. http://mathworld.wolfram.com/ TopologicallyTransitive.html, 2012. 